\title{
La Gouvernance Urbaine Dans les Espaces Marchands a Bafoussam (Ouest -Cameroun)
}

\author{
Flore Merline Megnijo Yemele, \\ Maurice Tsalefac, \\ Aristide Yemmafouo,
}

Centre d'Etudes et de Recherche en Espaces, Arts et Humanités (CEREAH), Département de Géographie, Université de Dschang, Cameroun

Doi:10.19044/esj.2020.v16n29p243 URL:http://dx.doi.org/10.19044/esj.2020.v16n29p243

\section{Résumé}

La question des espaces marchands est au cœur de la gestion des villes d'Afrique sub-saharienne. Ces espaces, dotés d'une forte valeur symbolique, remplissent une fonction économique et sociale cruciale. Leur gouvernance au Cameroun fait l'objet d'une exclusivité de l'Etat, acteur principal de la gestion des villes. L'explosion de l'effectif des commerçants rend les infrastructures marchandes largement insuffisantes. Commerçants de gros, détaillants, sauveteurs et autres colporteurs rivalisent dans l'occupation des espaces tandis que les communes d'arrondissement et la Communauté Urbaine se battent pour le contrôle de la gestion des marchés. L'objectif de cet article est d'analyser les jeux, enjeux et conflits qui émergent de l'intervention de différents acteurs sur les espaces marchands. L'hypothèse émise pose que les rivalités et les conflits entre acteurs de la gouvernance des espaces marchands tiennent à la fois des jeux d'intérêts personnels et collectifs souvent inavoués. Pour le vérifier, une enquête a été conduite auprès de 435 commerçants, 7 interviews ont été menées auprès des personnes ressources auxquelles se sont greffées des observations. Le traitement statistique et l'analyse des données quantitatives et qualitatives ont permis de comprendre que le désordre urbain dû à l'occupation anarchique de l'espace marchand à Bafoussam dévoile un déficit de gouvernance motivé par des intérêts divergents. La décentralisation qui aurait pu contribuer au développement de la ville a plutôt engendré des rivalités politiques et économiques. Les opérations de reconstruction des marchés érigées en mode de régulation de l'espace marchand par les CTD ${ }^{2}$ n'ont pas pu résoudre le problème de l'occupation anarchique desdits espaces par les commerçants. Il en ressort les comportements antisociaux, la mauvaise maintenance des infrastructures et la mauvaise image des espaces marchands.

\footnotetext{
${ }^{2}$ Collectivités Territoriales Décentralisées
} 
Mots clés : Gouvernance urbaine, gestion, espaces marchands, décentralisation, Bafoussam, Cameroun

\title{
Urban Governance in Commercial Spaces of Bafoussam (Western Region -Cameroon)
}

\author{
Flore Merline Megnijo Yemele, \\ Maurice Tsalefac, \\ Aristide Yemmafouo,
}

Centre d'Etudes et de Recherche en Espaces, Arts et Humanités (CEREAH), Département de Géographie, Université de Dschang, Cameroun

\begin{abstract}
The issue of commercial spaces is at the center of city management in sub-Saharan Africa. Such spaces, with high symbolic values, have a crucial economic and social function. Commercial spaces in Cameroon are exclusively handled by the State government, main actor of cities management. The exponential increase in number of merchants renders the available commercial infrastructures very insufficient. Dealers, retailers and other small scale traders compete for space while local government authorities and those in charge of cities ensure proper management of markets. The aim of this study is to evaluate the games, challenges and conflicts between different actors of commercial space. The hypothesis is that rivalries and conflicts between actors of commercial spaces governance represent both individual and collective games of interest, often denied. To test this hypothesis, an investigation was carried out on 435 merchants; 7 resource personnel's were interviewed and observations were made. Both quantitative and qualitative data were collected. Data management and Statistical analysis showed that urban disorder due to anarchic occupation of commercial space in Bafoussam reveals the lack of urban governance, motivated by divergent interests. Decentralization, which is expected to contribute to the development of cities, has instead brought political and economic rivalries. The reconstruction of markets by $\mathrm{DTC}^{3}$, in order to regularize/manage the commercial space, could not solve the problem of anarchic occupation of those
\end{abstract}

${ }^{3}$ Decentralised Territorial Authorithies 
spaces by merchants. This led to anti-social behaviours, poor maintenance of infrastructures and bad image of commercial spaces.

Keywords: Urban governance, management, commercial spaces, decentralization, Bafoussam, Cameroon

\section{Introduction}

Les espaces marchands sont des lieux de concentration, de créativité, de régulation sociale et d'intégration entre commerçants et non commerçants. Ce sont de puissants vecteurs d'organisation des territoires et des villes (Gaëlle \& Poisbeau, 2015). En Afrique, les grands marchés ont constitué, durant la période coloniale, des marqueurs et des outils d'aménagement des villes. Par la suite, ces marchés ont évolué et se sont transformés au gré des mutations des villes et des besoins d'approvisionnement des consommateurs urbains.

$\mathrm{Au}$ Cameroun, ils constituent des centres de création des richesses et un vecteur de croissance économique important. Commerçants fixes et ambulants se bousculent dans ces espaces cherchant à obtenir une place leur permettant de mener une activité lucrative.

A l'image des grandes métropoles, les marchés de Bafoussam constituent en effet une solution d'intégration économique et sociale des personnes les plus vulnérables, au travers d'une multitude d'emplois, majoritairement informels, de commerce ou de manutention. L'explosion de l'effectif des commerçants dans les marchés due à la crise d'emploi rend les infrastructures marchandes largement insuffisantes et difficiles à gérer. Ainsi, le débat sur la gestion et le fonctionnement de ces espaces doit être recentré ; car c'est par là que découle le désordre urbain se manifestant sur le plan économique à travers le secteur dit informel et se caractérisant par tout un réseau de petits métiers urbains qui se concentrent autour des grands marchés urbains (Assako, 1999). Les Maires, les cadres et les agents municipaux jouent leurs intérêts personnels ou corporatistes selon le temps et l'espace de l'action sur le terrain. Ainsi, intérêts économiques, voire politique se mêlent à la gestion des espaces marchands et créent une atmosphère de rivalité constante. Le même schéma de rivalité se constate entre les commerçants et les agents de terrain, et entre les commerçants eux-mêmes. Les épisodes de crise de gouvernance sont constants et montrent la nécessité d'élaborer une gouvernance durable des espaces marchands de Bafoussam. Partant de là, nous retenons la perspective selon laquelle les espaces marchands constituent des points très sensibles aux crises de gouvernance urbaine. Il se pose dès lors la question de savoir: comment s'exprime la demande de gouvernance urbaine dans les espaces marchands des villes moyennes du Sud fortement densifiée?

La gouvernance urbaine se manifeste à travers la constitution de coalitions formelles ou informelles, d'intérêts ou d'acteurs divers afin 
d'assurer la fourniture des biens et services. Cette option est considérée comme une stratégie de changement, de développement à la laquelle est invité le monde pour aller vers les espaces durables (Jacquier, 2008). Ici la volonté n'est pas de faire participer des acteurs privés, mais c'est plutôt de faire participer l'individu à la gestion des affaires de la collectivité comme l'affirme (Azeddine, 2010). (Bagnosco \& le Galès, 1997) renchérissent en soutenant que la coopération et l'accommodement entre des intérêts divers et conflictuels sont des processus continus. C'est ce que (Nahmias \& Hellier, 2012), appellent processus contractuels et négociés entre acteurs publics et acteurs privés au sens large et dans leur diversité. Mais, ces différents acteurs disposent d'un positionnement dissymétrique et d'un poids politique inégal dans les processus de décision ou de négociation. L'espace marchand en fait a une capacité spectaculaire à raviver les intérêts de ses acteurs ; néanmoins, au lieu d'être un espace propice pour la pratique de la gouvernance urbaine, il est resté un espace où se manifestent les égaux des acteurs. En effet, nous observons plutôt un processus de gouvernance qui se met en place entre des objectifs divergents et poussé par des intérêts, des façons de faire et des points de vue distincts, de la part des différents acteurs concernés. Ainsi donc, pour mieux appréhender la gouvernance des espaces marchands à Bafoussam, il conviendrait de présenter les enjeux et les conflits de la gouvernance desdits espaces. En explorant la diversité de ces espaces, il est présenté la méthodologie (I) comportant une présentation de la ville, Collecte et analyse des données ; les résultats (II) qui incluent les jeux et enjeux liés à l'occupation de espaces marchands et les rapports de forces dans la gouvernance de ces derniers. Une discussion (III) permet de relever l'originalité de la gouvernance des espaces marchands qui semble aujourd'hui faire cas d'école au service du rayonnement national et international de la ville.

\section{Méthodologie}

Dans cette partie de recherche, nous présenterons d'abord la ville de Bafoussam, ensuite la méthode de collecte des données et leur analyse, enfin les résultats.

\section{I.1. La ville de Bafoussam}

Bafoussam est la $3^{\mathrm{e}}$ ville du Cameroun après Douala et Yaoundé. Bafoussam est une ville du Cameroun, chef-lieu de la région de l'Ouest, cheflieu du département de la Mifi et l'un des trois arrondissements de ce département. Elle a été érigée en Communauté Urbaine de Bafoussam en 2008. Située en pays Bamiléké, Bafoussam est à la fois ville et village du peuple du même nom. Sa population est intime d'environ de 347517 
habitants $(2008)^{4}$; la cité est à la tête de 31 villes que constitue le réseau de la région de l'Ouest. Cette région se classe parmi les plus densément peuplé du Cameroun (400hbts $/ \mathrm{km} 2)$. Situé entre le $10^{\circ} 20^{5}$ et $10^{\circ} 35^{5}$ de longitude Est et entre le $5^{\circ} 25^{\prime}$ et $5^{\circ} 35^{\prime}$ de latitude Nord, Bafoussam s'étend sur une superficie de 5000 ha seulement (Ndoki et al 2016).

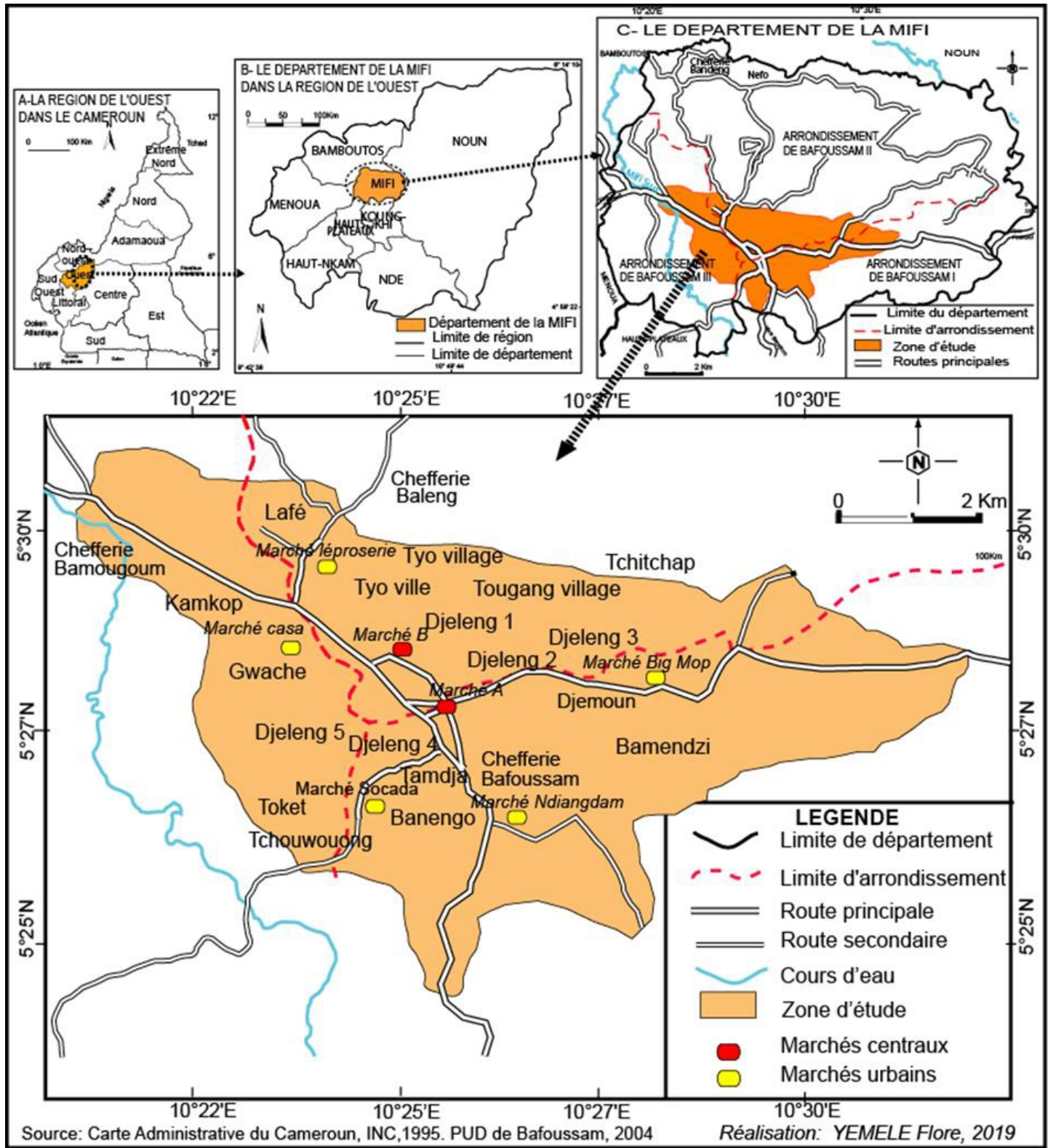

Figure 1. Situation de la zone de recherche

${ }^{4}$ Décret no 2008/025 du 17 Janvier portant création de la Communauté Urbaine de Bafoussam, In Cameroon Tribune, no 9018 du vendredi 18 Janvier 2008, p5. 
Sa position stratégique au centre de la région administrative de l'Ouest Cameroun la place au cœur des activités économiques. Son statut administratif explique d'une part son évolution très rapide puisqu'elle draine une partie de la population des autres villes du pays et même des pays voisins. Sa position centrale dans la région de l'ouest Cameroun lui permet d'avoir une situation privilégiée. Cette évolution spatiale et démographique très rapide lui vaut d'être confronté à des problèmes de gouvernance urbaine. L'attitude passive des autorités en charge de leur administration a laissé ainsi la liberté aux commerçants d'improviser des commerces partout où bon leur semble et au gré de l'affluence des clients. Ceci rend le paysage urbain désordonné et permet de faire une analyse de la gestion dans cette ville.

\section{I.2. Collecte et analyse des données}

Les résultats de recherche ont été obtenus à partir d'un travail de recherche sur le terrain. Une enquête auprès de 435 commerçants et 7 interviews auprès du Délégué du Gouvernement, les maires d'arrondissements, les délégués des commerçants, et le président de l'association des commerçants pendant les années 2017-2018 ont permis de collecter les données utilisées dans ce travail. Une approche empirique qualitative et quantitative a été adoptée. Après observation des différents espaces, une étude a été conduite dans les marchés de Bafoussam. L’objectif des interviews était d'une part de recueillir des données qualitatives relatives aux politiques de gestion des espaces marchands à Bafoussam et d'autre part de relever les différents conflits qui s'y trouvent.

Afin de mieux faire ressortir le caractère pluriel des espaces marchands à Bafoussam lors des enquêtes par questionnaires, nos enquêtes ont ciblé 3 zones : La première zone est le centre-ville de Bafoussam où se trouve le marché A et B. le centre-ville est en effet un observatoire pour mieux cerner les enjeux autour de la gestion de l'espace urbain. Les conflits y sont récurrents entre commerçants et autorités municipales animées par le désir de décongestionner les rues et moderniser la ville. Les commerçants rejettent toute idée de déguerpissement les empêchant d'accéder à la clientèle qui les fréquente régulièrement. La deuxième zone est constituée du marché Casablanca, elle a la particularité d'être un espace commercial destiné à la vente du bétail et des chaussures. Mais ce marché reste encore inoccupé en grande partie et les commerçants préfêrent retourner dans leurs sites initiaux. La troisième zone concerne les marchés périphériques tel que le marché $\mathrm{C}$ où, suite à la loi de décentralisation, les conflits sont nés entre le Délégué du Gouvernement et les maires à cause de la non maitrise de cette loi. L'échantillonnage aléatoire systématique a été utilisé pour choisir les commerçants à enquêter dans chaque zone et selon l'activité homogène. Le pas de sondage dégressif de 4,2, 1 a été retenu selon la densité des 
commerçants sur les espaces. Nous avons remplis nos questionnaires en présence des commerçants. Le traitement des données quantitatives a été fait à l'aide de SPSS et restitués sous formes de tableaux descriptifs et celles qualitatives ont fait l'objet d'analyse thématique.

\section{Résultats}

\section{II.1. Jeux Et Enjeux De La Gouvernance Des Espaces Marchands De Bafoussam}

\section{II.1.1. Jeux et enjeux socio-économiques}

\section{L'espace marchand: véritable source de recette pour la Commune}

La décennie 1990/2000 a été marquée au Cameroun par une avancée importante dans le cadre de la décentralisation. Les collectivités locales ont été dotées d'une personnalité juridique et de l'autonomie financière bien que l'Etat exerce toujours un contrôle de légalité. Les enquêtes effectuées dans les services de recette (chef service du budget) nous permettent de constater la situation financière de la Communauté Urbaine de Bafoussam au cours des dernières années (graphique 2).

Figure 2. Evolution des Recettes moyennes de la CUB en FCFA entre 2011 et 2016

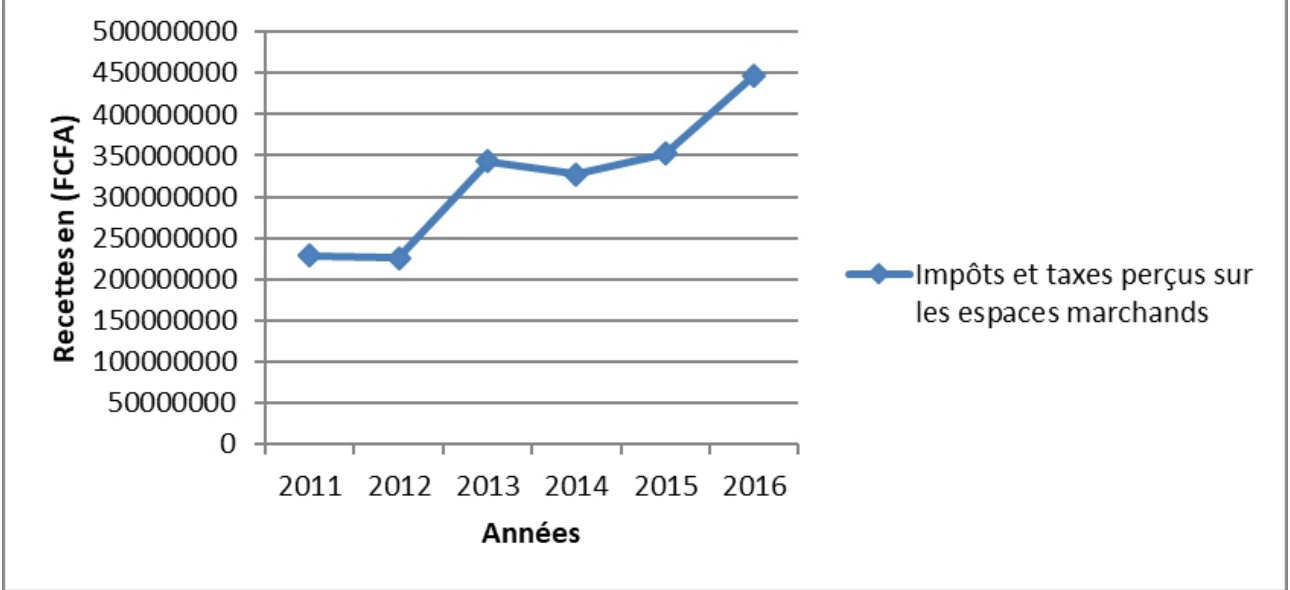

Source : données du service d'assiette de la commune de Bafoussam, enquête de terrain, Novembre 2017

A la lecture de cette figure, nous constatons que les recettes ont évolué de manière considérable de 2011 à 2016. A la faveur des conflits entre les communes d'arrondissements et communauté urbaine, on est passé de 228475 066 FCFA au cours de l'exercice 2011 à 446858141 FCFA au cours de l'exercice 2016 ; ce qui correspond à $11,87 \%$ et $23,11 \%$ du rapport des recettes totales de la CUB. En plus, le déficit fiscal de la communauté a été constaté dans les années antérieures. Héritant de ce déficit, l'actuel exécutif a besoin de revitaliser ses ressources financières pour atteindre ses objectifs. Il faut donc créer des infrastructures qui permettent de faire des entrées à la 
communauté urbaine. L'enjeu, est de stimuler les activités économiques dans la ville. C'est ce qui explique la construction des boutiques dans les marchés, tout au long de la ville et autour des agences de voyage. Ces entités contribuent indéniablement à l'amélioration des recettes communales. Le Maire recherche en effet un cadre de rentabilité maximale car le décret du Premier Ministre signé le 20 Décembre 2002 autorise les communes à percevoir non seulement un loyer dont le montant oscille entre 5000 FCFA et 200000 FCFA par mois dans les marchés pour la location des boutiques couvertes, mais également des droits de place sur ces marchés dont le tarif maximum est de 250 frs CFA par jour pour tout produit commercialisé. Ainsi parmi les commerçants enquêtés 207 personnes soit 49,64\% déclarent payer la location qui varie de [-5000 ; +20000 [tous les mois. Le loyer est le secteur par lequel la Communauté réalise des recettes par excellence ; en 6 ans, elle perçoit 1040767068 de FCFA dont 244629241 en 2016. La prise des commandes par le Délégué du gouvernement entraine ipso facto l'amélioration des recettes ; Ceci est dû à la multiplication des stands dans les espaces marchands. en 2011 par rapport aux années antérieures, La chute a été due aux mouvements d'humeur des commerçants qui se sont opposés à l'application de la loi règlementant les taux de loyers dans tous les marchés communaux du pays, et qui ne se sont pas acquittés des frais du loyer. Le Droit de place a considérablement baissé à cause des opérations de déguerpissement réalisé dans la ville ; elle quitte de 22537060 FCFA en 2011 à 17528900FCFA en 2016.

Les marchés constituent à priori, des gisements importants de ressources au travers des loyers, des droits de place, l'OTVP ${ }^{5}$ et de la patente. Dans la réalité, les marchés ne fournissent pas les recettes escomptées, qui ne dépassent que très rarement $10 \%$ des recettes courantes des grandes villes d'Afrique subsaharienne (Wilhelm, 1994). Par exemple, les comptes administratifs de la commune de Ouagadougou montrent que les marchés et les gares routières alimentent le budget de la ville à hauteur de FCFA 40 à 50 millions (les marchés représentant en FCFA 12 millions). Ce montant équivaut à cinq pour cent des recettes de fonctionnement réelles de la commune. Les potentialités financières prévues et disponibles des communes ne leur permettent pas de faire de réalisations importantes. Les grands investissements restent du domaine privilégié de l'État; La notion de l'autonomie de gestion reste à confirmer dans les faits (Tsobzé et al. 2007). On constate donc que les logiques d'action des différentes autorités révèlent à la fois une volonté d'organiser les espaces marchands et celle d'en tirer profit. L'espace marchand a aussi un impact sur les revenus des commerçants.

\footnotetext{
${ }^{5}$ Occupation Temporaire de la Voie Publique
} 
- L'espace marchand: source de revenu pour les commerçants.

L'espace marchand et les services existants sont insuffisants dans la ville de Bafoussam et beaucoup de commerçants occupent donc les trottoirs autour des marchés pour mener leur activité. On y distingue les calls boxeurs, les restaurateurs, les couturiers, les fripiers, les vendeurs de vivres appelés «bayam sellams», les offres de services. Ces commerçants sont classés en 3 secteurs selon leurs activités et leurs revenus dans la figure 3 ci-après.

Figure 3. Evolution des revenus moyens des enquêtés dans trois secteurs d'activités de 2014

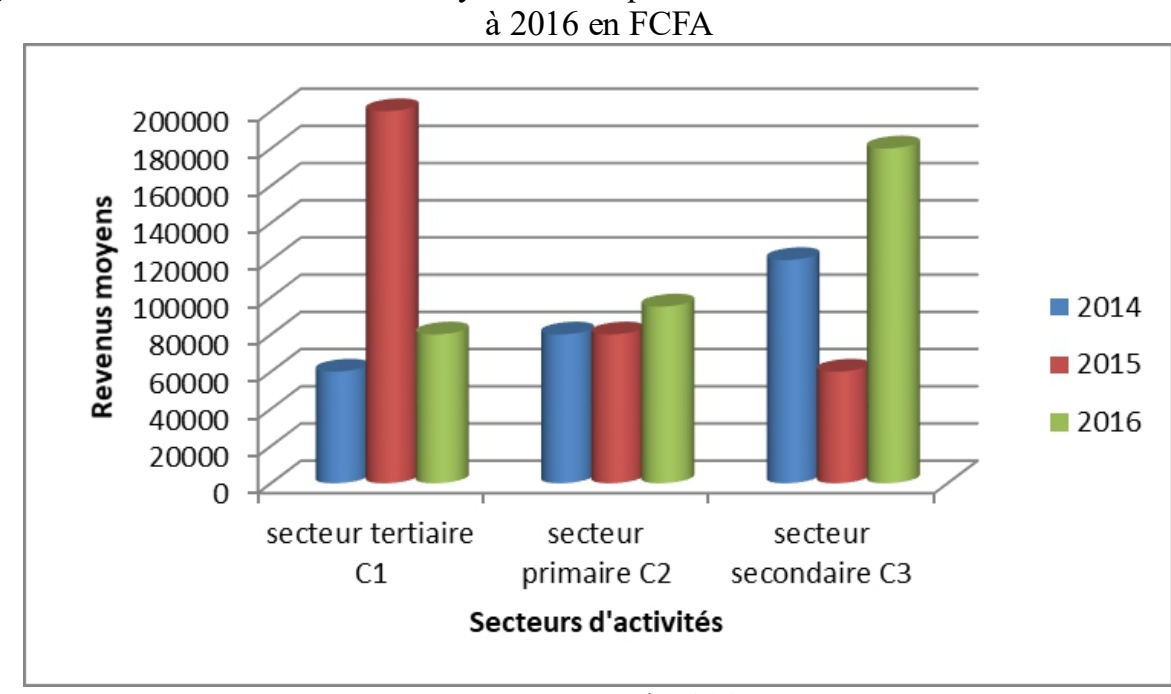

Légende :

Source : Yemele, 2017

$\mathrm{C} 1$ : commerçants du secteur tertiaire : call box ${ }^{6}$,

$\mathrm{C} 2$ : commerçants du secteur primaire : vendeurs des vivres ${ }^{7}$ et

C3 : commerçants du secteur secondaire : les fripiers ${ }^{8}$

(C1 et $\mathrm{C} 3)$; quant à $\mathrm{C} 2$, elle est descendante. La croissance ou la décroissance des revenus indique respectivement l'augmentation et la baisse des chiffres d'affaire. D'après les témoignages des commerçants, généralement l'augmentation des revenus est liée à l'augmentation de la population dans la ville et la baisse est relative à la multiplication exagérée des

${ }^{6}$ Le secteur tertiaire recouvre un vaste champ d'activités qui s'étend du commerce à l'administration, en passant par les transports, les activités financières et immobilières, les services aux entreprises et services aux particuliers, l'éducation, la santé et l'action sociale ; nous nous en tenons sur les transporteurs

${ }^{7}$ Secteur primaire ; (nom masculin) le primaire domaine des activités productrices de matières non transformées : agriculture, pêche, mines...

${ }^{8}$ Le secteur secondaire correspond aux activités liées à la transformation des matières premières, qui sont issues du secteur primaire. Il comprend des activités aussi variées que l'industrie du bois, l'aéronautique et l'électronique, le raffinage du pétrole, la production industrielle, la construction. 
activités du même secteur sur le marché. A cette multiplication, cause de la baisse des revenus, se greffe aussi la concurrence déloyale. Ce phénomène traduit une sorte d'élasticité de la demande par rapport à l'offre, dans la mesure où une variation des revenus est liée à une variation relative de la demande.

Ceci illustre davantage et de façon claire le caractère attractif des marchés. Par rapport aux autres années, l'année 2016 représente le pic dans l'évolution des revenus $\mathrm{C} 1$ et $\mathrm{C} 2 ; 2016$ représente celui de $\mathrm{C} 3$. Les activités des secteurs primaire, secondaire et tertiaire ont contribué respectivement pour $23,2 \%, 27,4 \%$, et $36,4 \%$ au $\mathrm{PIB}^{9}$ de $2000 / 2001$ et à $59 \%, 12 \%$, et $27 \%$ à l'emploi. Le secteur économique informel est largement prédominant au regard du nombre d'emplois offerts. Il héberge plus de $2 / 3$ des emplois, le tiers restant se répartissant entre fonction publique et secteur « moderne » de l'entreprise. Cette variation de revenus s'explique par le fait que tous les commerçants n'exercent pas la même activité ; par la situation des stands : certains stands occupent des espaces dont l'accès est facile pour les clients, et aussi par les politiques commerciales de chaque commerçants; certains préfèrent vendre leurs produits moins chers et rapidement que de vendre chers pour une longue durée. Tout ceci montre que ces derniers font cortège à la concurrence déloyale réprimée par la loi N 90/031 du 10 Août 1990 régissant l'activité commerciale au Cameroun.

Sur le plan social; la gouvernance urbaine à travers l'accès aux services sociaux de base : eau, électricité, etc.... Les points d'eau sur les marchés sont inexistants ou ne fonctionnent plus. L'approvisionnement des usagers se fait par porteurs d'eau ou en achetant l'eau de branchements privés. La diversité des situations rencontrées entraîne de fortes variations du prix d'achat de l'eau (voir photo ci-dessous).

${ }^{9}$ Produit Intérieur Brut 
Figure 4. Point d'eau installée par un particulier au marché A

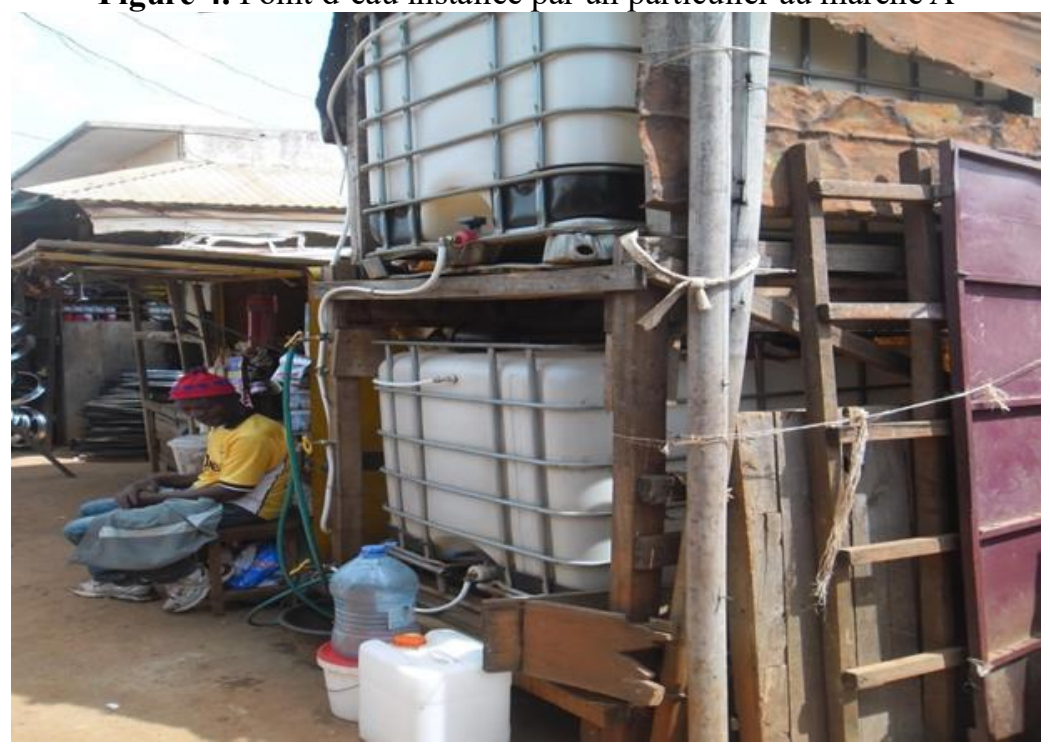

CYemele, 2017

Ce point d'eau aménagé par un particulier est une source d'approvisionnement du marché A; le propriétaire en a fait une activité génératrice de revenus et il compte installer ces bonbonnes dans d'autres secteurs du marché pour pouvoir embaucher les jeunes et réduire le taux de chômage.

Sur tous les marchés, l'emploi d'un gardien est la règle pour les commerçants installés dans une boutique ou dans un hangar, et est même assez fréquent chez les vendeurs disposant d'une table. Le coût du gardiennage est très souvent partagé entre plusieurs vendeurs. Le montant de la rémunération varie suivant la valeur des marchandises à garder et les services complémentaires qui peuvent lui être demandés. Ce coût varie de $500 \mathrm{f}$ à $1000 \mathrm{f}$ par semaine avec en moyen 2 à 3 gardiens par secteur. Ce gardiennage privé semble insuffisant et dans certains marchés comme les marchés B et Casablanca presque tous les commerçants se plaignent du développement des vols, de nuit comme de jour. L'amélioration des infrastructures permet de rendre les marchés plus attrayants et à l'abri des cambrioleurs.

\section{II.1.2. Jeux et enjeux fonciers}

L'accès à l'espace marchand se fait selon diverses modalités. L'on peut distinguer plusieurs modes d'accès à l'espace (récapitulé comme suit : les propriétaires $23 \%$, les locataires $27 \%$, la sous location $24 \%$, l'accès gratuit $17 \%$, autres $9 \%$.) 
Figure 5. Les modes d'accession à l'espace marchand à Bafoussam

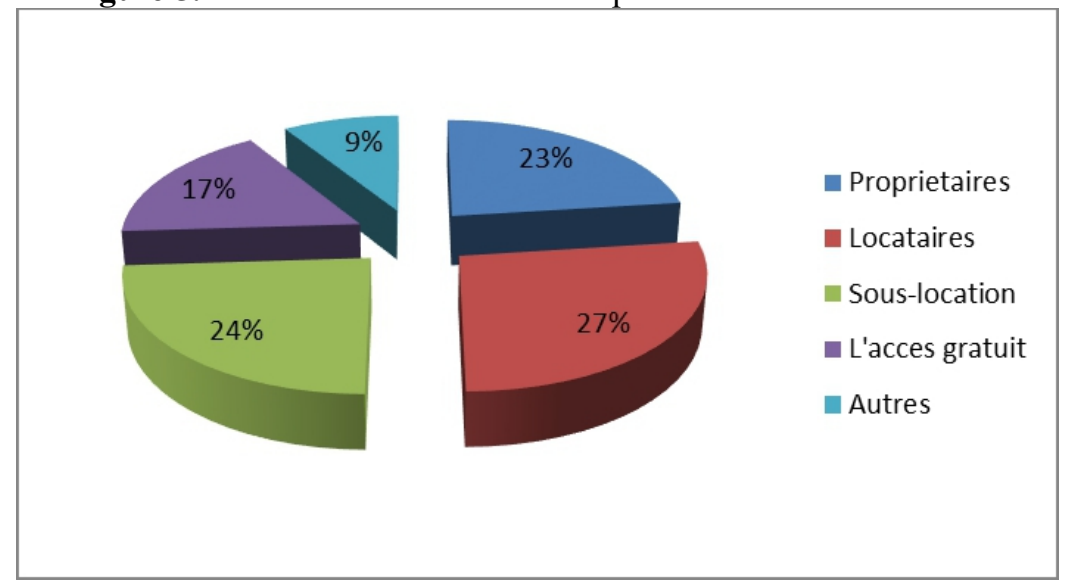

Source : Yemele, 2017

Les locataires sont ceux qui louent directement à la Communauté Urbaine. Ils sont plus nombreux et représentent $27 \%$ de l'effectif enquêté. Ceux qui font la sous location représentent 24\%. Leur taux est élevé par rapport à celui des propriétaires parce que ces derniers possèdent plusieurs boutiques qui les permettent de faire la sous-location. Les propriétaires sont ceux qui ont acquis l'espace soit par achat, soit par héritage. Ils représentent 23\% des commerçants. Là encore la reconstruction de certains secteurs des marchés et la position de capital économique de l'Ouest sont des facteurs à prendre en compte car ils ont contribué à la hausse de la demande de l'espace de vente. Cela a permis la multiplication de ces espaces. Il ressort de ce tableau que l'accès personnel ou gratuit représente $17 \%$ des enquêtés montrant l'état de pauvreté de la population car incapable de louer un stand. On peut observer que les logiques d'action des commerçants démontrent une volonté de garantir le contrôle et la possession de leur place de vente à travers le contrôle d'accès à ces places par un système de transactions, ainsi que pour assurer la sécurité des lieux ; car obtenir une place sur le marché est un obstacle bien plus grand que d'obtenir des marchandises (Lewis \& al 1976).

Les commerçants ne sont pas les seuls demandeurs d'espace. La liste des acteurs qui exigent ce droit est longue: déchargeurs, porteurs, pousseurs, vendeurs de repas ou d'eau, fabricants d'étals ou de paniers, gardiens, locataires d'espaces de magasins ou de la cour familiale. Ainsi les petits commerçants cherchent de l'espace pour étaler leurs produits tandis que les transporteurs cherchent de grands espaces pour stationner les cars. Ce qui entraîne la dégradation de l'environnement par des encombrements et voire même par des pollutions. 


\section{II.1.3. Jeux et enjeux écologiques}

L'environnement des espaces marchands est caractérisé par l'insalubrité, la promiscuité, l'inconfort et l'accessibilité difficile. Sans système de régulation, les allées sont encombrées et empêchent toute évacuation rapide des ordures; des dysfonctionnements dans la collecte des déchets aboutissent rapidement à l'apparition des dépotoirs représentant un danger sanitaire pour les commerçants et leurs clients. (voir photo ci- après).

Figure 6. Planché montrant l'inaccessibilité et l'insalubrité aux marché casa et marché B

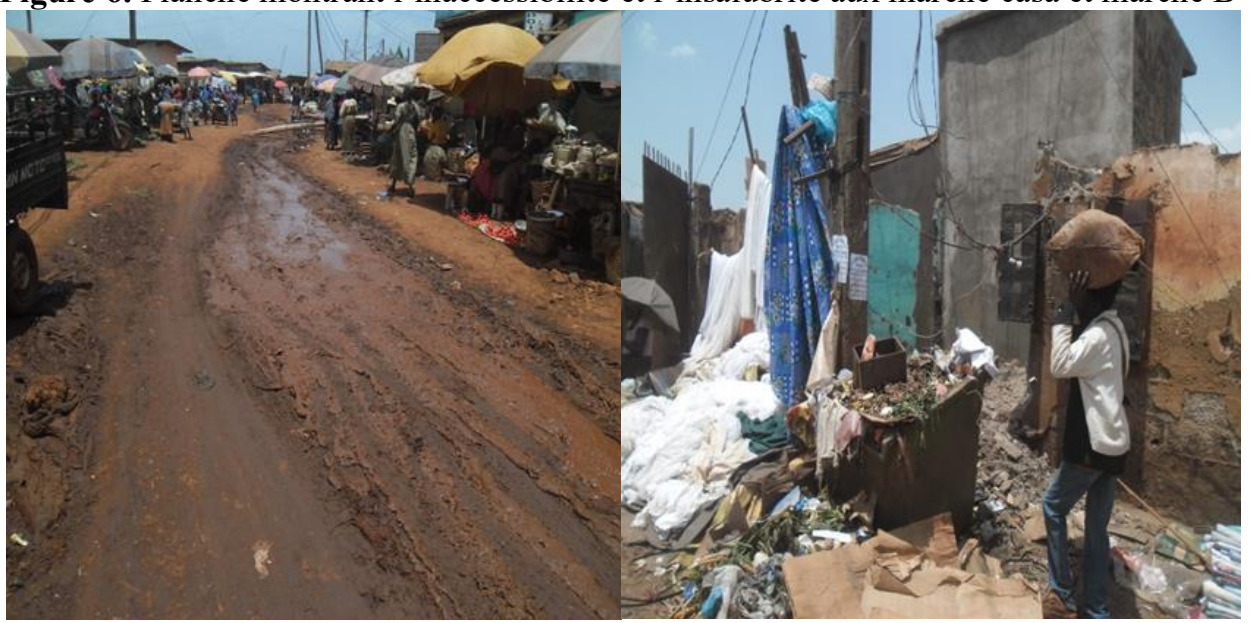

(C) Yemele, 2017

Ces deux photos présentent l'inaccessibilité et l'insalubrité dans les marchés à Bafoussam. Ainsi Ces caractéristiques propres aux villes du tiers monde, se retrouvent aussi à Bafoussam où l'occupation du sol précède l'aménagement. Malgré le relief, la qualité du sol, les passages et les routes non entretenues, l'on constate la prolifération de nombreux espaces marchands dont l'utilisation présente un inconfort permanent surtout en saison pluvieuse. La pluie occasionne à cet effet de grandes nappes d'eau stagnantes qui jonchent sur le sol imperméable du fait de sa nature argileuse, rendant les couloirs et les routes presque impraticables à cause de la boue rependue un peu partout. L'exemple des marché A, B, Casablanca ; de la gare principale Ndiangdam, du point de chargement marché "B" en constituent une parfaite illustration.

Depuis les années 2000, les problèmes liés à l'organisation de ces espaces marchands préoccupent les administrateurs de la ville. 


\section{II.2. Les Conflits Liés à La Gouvernance des Espaces Marchands à Bafoussam \\ Un chevauchement de compétences entre les communes d'arrondissement et la communauté urbaine}

Dès la publication du Décret N²015/1375/PM du 08 Juin 2015 fixant les modalités d'exercice de certaines compétences transférées par l'Etat aux Communes en matière de commerce; le Maire de la commune d'arrondissement a interprété le contenu du décret de façon erronée. Selon lui, l'Etat venait de leur transférer la gestion de tous les marchés et de tous les espaces marchands ce qui devrait améliorer ainsi leurs recettes. Or ils oublient que l'entretient de ces espaces incombe à la Communauté Urbaine qui a besoin de ces recettes pour accomplir ses tâches consistant en l'éclairage public, le paiement des factures y relatives, le balayage, l'enlèvement des ordures ménagères, l'entretien de la voirie urbaine par le reprofilage des routes dans les quartiers, la construction des ponts et dalots. Font également partie des obligations de la Communauté Urbaine, les charges incompressibles liées au salaire du personnel, au fonctionnement, à la dotation trimestrielle de fonctionnement à des communes d'arrondissements. Ces administrateurs municipaux se fondent ainsi sur le fait que le transfert dont il s'agit est destiné aux Communes d'arrondissement seulement. Pourtant, la constitution du Cameroun prévoit deux types de collectivités territoriales décentralisées à savoir les Régions et les Communes. Au Cameroun il existe des communautés urbaines et des communes d'arrondissements. Les premières sont administrés par des DG de nos jours appelés supers maires et les secondes par les maires (maire d'arrondissement). C'est ces deux types d'exécutifs municipaux qui mènent une lutte pour contrôler la gestion de la ville dans le cadre de la décentralisation, interprétant maladroitement les textes règlementant ce domaine. Ce faisant, ils ont procédés à des recensements sans base légale dans les marchés et ont identifié les locataires en attendant de donner l'assaut final par la prise de possession des lieux. Ils ont exigé aux locataires des droits de place sur les marchés (loyers) entre leurs mains en rappellent aux commerçants le paiement dans les marchés relèvent de leur compétence. Cette situation intéresse particulièrement les locataires indélicats qui ne voudraient s'acquitter du loyer, ceci pour faire échec aux actions municipales.

Certaines Communes de la Mifi se sont permis de convoquer une session extraordinaire du Conseil Municipal au cours de laquelle, elles ont pris des résolutions habilitant leur Maire à prendre possession de tous les marchés et espaces marchands de leur territoire de compétence pour les gérer. Malheureusement pour eux, le Préfet, Autorité de Tutelle avait rejeté la délibération issue des travaux dudit conseil. C'est dans cette logique qu'un groupe de femmes récalcitrantes, ayant occupé de force certaines boutiques en face de la boucherie du marché central de Bafoussam ont catégoriquement 
refusé ; délibérer les stands irrégulièrement occupés disant que « la gestion des marchés ne relève plus de la compétence du Délégué du Gouvernement mais plutôt aux maires des Communes d'Arrondissement ».

Dans la première semaine du mois de mars 2016, certains agents communaux ont sillonné le marché A de Bafoussam en disant aux commerçants : «méfiez-vous. Ne payez rien à la Communauté Urbaine. Celui qui paie aux mains non autorisées son loyer le paiera deux fois ». Face à cette situation la CUB a saisi le MINATD ${ }^{10}$ pour une clarification des données, en s'appuyant sur la base de l'article 110 de la loi N²004/018 du 22 Juillet 2004 fixant les règles applicables aux communes. Cette loi précise entre autres les compétences transférées aux Communautés Urbaines à compter de leur création, celle relative à « la gestion des marchés ». Les marchés dont les Communes d'Arrondissement réclament la gestion relèvent de la compétence de la Communauté Urbaine. A cause de cette confusion, une mission interministérielle de haut niveau composé des représentants du MINATD et du Ministère du Commerce a été dépêchée à Bafoussam le 08 Mars 2016. Au cours d'une séance de travail qui s'est tenue dans la salle des actes de la Préfecture de la Mifi à Bafoussam à laquelle ont; pris ; part : le Préfet de la Mifi, le Délégué du Gouvernement, les Maires des Communes d'Arrondissement de la Mifi et leurs Adjoints, le Secrétaire Général de la CUB et ceux des Communes d'Arrondissement. Il a été passé au crible le contenu du décret $\mathrm{N}^{\circ}$ 2015/1375/PM du 08 Juin 2015 fixant les modalités d'exercice de certaines compétences transférées par l'Etat aux Communes en matière de commerce. Tous ont compris et suivi attentivement comme dans un amphithéâtre, les explications des experts de la mission interministérielle. Il est ainsi ressorti que les marchés et les espaces marchands de la ville de Bafoussam sont la propriété de la Communauté Urbaine et comme tel, gérés par le Délégué du Gouvernement. Les Communes d'Arrondissement peuvent dans le cadre de l'amélioration des conditions de vie de leurs populations, détecter dans leur territoire respectif, les zones de grands rassemblements, les zones d'échanges et y construire des marchés de proximité ou des marchés périodiques.

Ainsi, nous relevons qu'il existe un chevauchement de compétences entre les communes d'arrondissement et les communautés urbaines. Cela est dû à un manque de réalisme juridique du législateur dans l'attribution des compétences spécifiques à chacune des institutions locales. Certains spécialistes de droit public pensent en effet que cette situation cause polémique pour des raisons de l'existence d'une institution locale, créée à cet effet pour contrôler l'opposition qui gagnait de plus en plus le terrain dans la gestion locale et municipale au Cameroun. Cette analyse trouvait son

\footnotetext{
${ }^{10}$ Ministère de l'Admistration Territoriale et de Décentralisation
} 
fondement dans les années 1992 avec la percée spectaculaire de l'opposition politique dans les institutions locales ; mais aujourd'hui où le parti au pouvoir a conquis toute l'échelle locale, l'on s'interroge encore sur l'existence et la suprématie de cette institution sur les communes supposées avoir la maitrise de la gestion locale au Cameroun. Tout compte fait, que ce soit les communes urbaines, d'arrondissement ou les communautés urbaines, l'on s'aperçoit du rôle indispensable qu'elle joue dans la gouvernance locale et au développement durable de nos villes. Cependant, pour être efficace et efficient, le développement urbain doit s'accompagner des moyens dans la réalisation des projets sociaux et urbains durables.

\section{Conflit consécutif à l'augmentation des impôts}

Les commerçants du marché central ont mené une grève en 2017 évoquant les pressions fiscales et les abus des agents d'impôts de la communauté urbaine et de la mairie de Bafoussam $1^{\text {er }}$. "Jusqu'à 11 heures le 4 avril 2017, toutes les boutiques et magasins étaient fermés. Ceci à cause de l'augmentation des impôts sans justificatifs affirme le délégué des commerçants ». En effet, la création de nouveaux impôts a conduit à la fermeture de nombreuses boutiques et autres magasins dans les principaux marchés de la ville. Depuis quelques mois, une tension palpable s'est installée entre les commerçants et les partenaires administratifs traditionnels. Un commerçant allègue volontiers :

\section{Encadré1 : comparaison d'impôts}

«Je paye 130000 FCFA d'impôts par an alors que les commerçants de même niveau que moi ne payent que 60000 FCFA à Douala.».

\section{Extrait d'un entretien avec un commerçant Décembre 2017}

En plus de cela, ces commerçants évoquent des cas d'arnaques orchestrées par des agents des impôts, en service dans la Communauté d'arrondissement de Bafoussam $1^{\mathrm{er}}$. Dans la même lancée, la Division régionale des Impôts a initié l'instauration d'un recensement qui a abouti à l'adressage des boutiques et magasins des marchés de la ville. Devant le refus des commerçants de se soumettre au nouveau recensement, l'administration des impôts a procédé aux scellés de nombreuses boutiques et magasins, ainsi qu'a plusieurs perquisitions. Par contre, du côté de l'administration, le refus de payer les impôts et taxes est imputé à de nombreux commerçants. 
Encadré2 : délocalisation des magasins.

«La tendance chez les commerçants est de plus en plus à la délocalisation des magasins de stockage vers des zones périphériques pour organiser la fuite devant les impôts et se soustraire à leur payement»

Extrait d'un entretien avec un Délégué commerçant Décembre 2017

À en croire cette source proche de l'administration des impôts, des investigations ont abouti à la découverte de magasins et entrepôts logés dans les quartiers et domiciles de certains commerçants qui

\section{Encadré3 : fausse déclaration}

"les commerçants déclarent souvent jusqu'à mille fois moins leurs chiffres d'affaire à la fiscalité ».

Extrait d'un entretien avec le responsable du Bureau des espaces marchands Novembre 2017

Les marchés urbains sont généralement considérés par les pouvoirs publics comme une source de revenus mais, souvent, ces fonds ne sont pas réinvestis dans l'entretien des infrastructures pour l'amélioration des services. Cela amène les commerçants à considérer les taxes de marché non justifiées et sans sens, entrainant ainsi des conflits sociaux au moment de l'augmentation de leurs taux. Les commerçants se plaignent du manque de système de surveillance approprié. Ainsi les vols occasionnent des dépenses chez les commerçants obligés de louer des espaces de stockage sécurisés à proximité des marchés.

Conflit entre les commerçants eux-mêmes (rapport entre commerçants riches et commerçants à revenus faibles à propos des projets de reconstruction $d u$ marché $\left.e^{\prime \prime} A^{\prime \prime}\right)$

Le projet de reconstruction du marché "A" a créé une complexité des rapports que les commerçants entretiennent entre eux. Les différentes catégories de commerçants s'unissent et se désunissent dans la lutte contre les municipalités pour préserver leurs intérêts : payer une redevance peu élevée pour conserver leurs places. Dans ce but, les commerçants autochtones se battent contre une appropriation de leur place par des commerçants allogènes qui auraient certainement de moyens suffisants pour payer de nouvelles taxes. La problématique de la différenciation entre autochtones/allogènes est ravivée par l'utilisation de la crainte de l'autre, l'étranger ${ }^{11}$. Ce dernier se définit

${ }^{11}$ Ce dernier est défini comme celui qui constitue une menace dans l'exercice de l'activité commerciale et de l'appropriation d'un espace 
comme étant celui qui constate l'appropriation d'espace car disposant des moyens suffisants par rapport à l'autochtone. Face aux dangers de la perte d'influence de gros commerçants locaux, on assiste alors à un véritable repli des commerçants locaux vis-à-vis aux étrangers. Cela conduit « à une recomposition de l'idée d'appartenance et de citoyenneté», lourde de menaces, mais qui demeure probablement ouverte à d'autres possibilités historiques, compte tenu de la complexité des mouvements sociaux (Bayart, \& al 2001). Il sera donc impératif d'instaurer un dialogue local pour gérer la situation.

\section{Conflit entre police municipale et commerçants}

Les politiques de délocalisation de certains commerçants ont entrainé le conflit entre la police municipale et ces derniers. En fait, ces commerçants affirment gagner au moins 2000f CFA par jour et que la pression permanente exercée par la police municipale de manière improvisée fait chuter leurs revenus de 30 à $60 \%$, cela les place en position inconfortable. «Non seulement vos bénéfices diminuent, mais votre train de vie baisse sensiblement. Par conséquent vous perdez du pouvoir sur le site». Ici il faut chercher à rompre avec la culture du chef qui distribue ses prébendes afin d'assurer l'appropriation du nouveau marché par les acteurs concernés à travers l'intermédiation sociale. Cette intermédiation vise à instaurer le dialogue entre les parties prenantes (commerçants, municipalités), de manière impartiale et continue (Michelon, 2008, P1). Cet appui externe doit préparer la gestion future et faciliter la concertation entre les acteurs. Il doit également permettre d'appuyer la mise en place d'un nouvel «arrangement» entre les parties.

\section{Conflit entre les agents de la CUB, les commerçants et particuliers du marché mondial}

Le marché mondial est un espace partagé entre le privé et le public: d'un côté c'est l'espace public et de l'autre côté c'est un espace aménagé par les particuliers possédant des titres fonciers. Dans cet espace, le milieu (la route) est occupé par les commerçants dont certains déclarent payer leurs droits aux particuliers. Or les agents de la CUB passent avec les tickets les obligeant de payer l'OTVP. Ces derniers refusent et cela engendre des conflits. Jusqu'aujourd'hui ces conflits ne sont pas solutionner car les agents municipaux en ont fait un business les permettant de compléter leurs salaires. Les recettes qu'ils parviennent à faire sur cet espace ne sont pas verser en totalité et la CUB n'est pas au courant ; Pourtant elle en a besoin pour réaliser ses projets.

Les conflits entre le délégué du gouvernement auprès de la Communauté urbaine de Bafoussam et les différentes municipalités ; les problèmes entre la CUB et certains propriétaires de boutiques dits privés dans la capitale économique de l'Ouest doivent complètement finir. Selon l'article 
115 de la loi n²009/019 du 15 décembre 2009 portant fiscalité locale, chaque collectivité territoriale gère directement les marchés qu'elle a aménagés et qui sont sa propriété. La Communauté Urbaine est l'exécutif communautaire chargé de superviser et de réglementer la création, la délocalisation ainsi que la gestion des marchés qui lui appartiennent. Les communes peuvent en accord avec la Communauté urbaine, exercer ces mêmes prérogatives de même qu'elles gèrent les marchés qui leur appartiennent. Les commerçants sont des opérateurs économiques dont toute implication dans la gestion de l'équipement public marchand, vu le conflit d'intérêt probable, ne peut être que préjudiciable à l'ordre des choses. Ces enjeux et conflits seront représenté sur le schéma suivant :

Figure7. Schéma de la gouvernance des marchés à Bafoussam

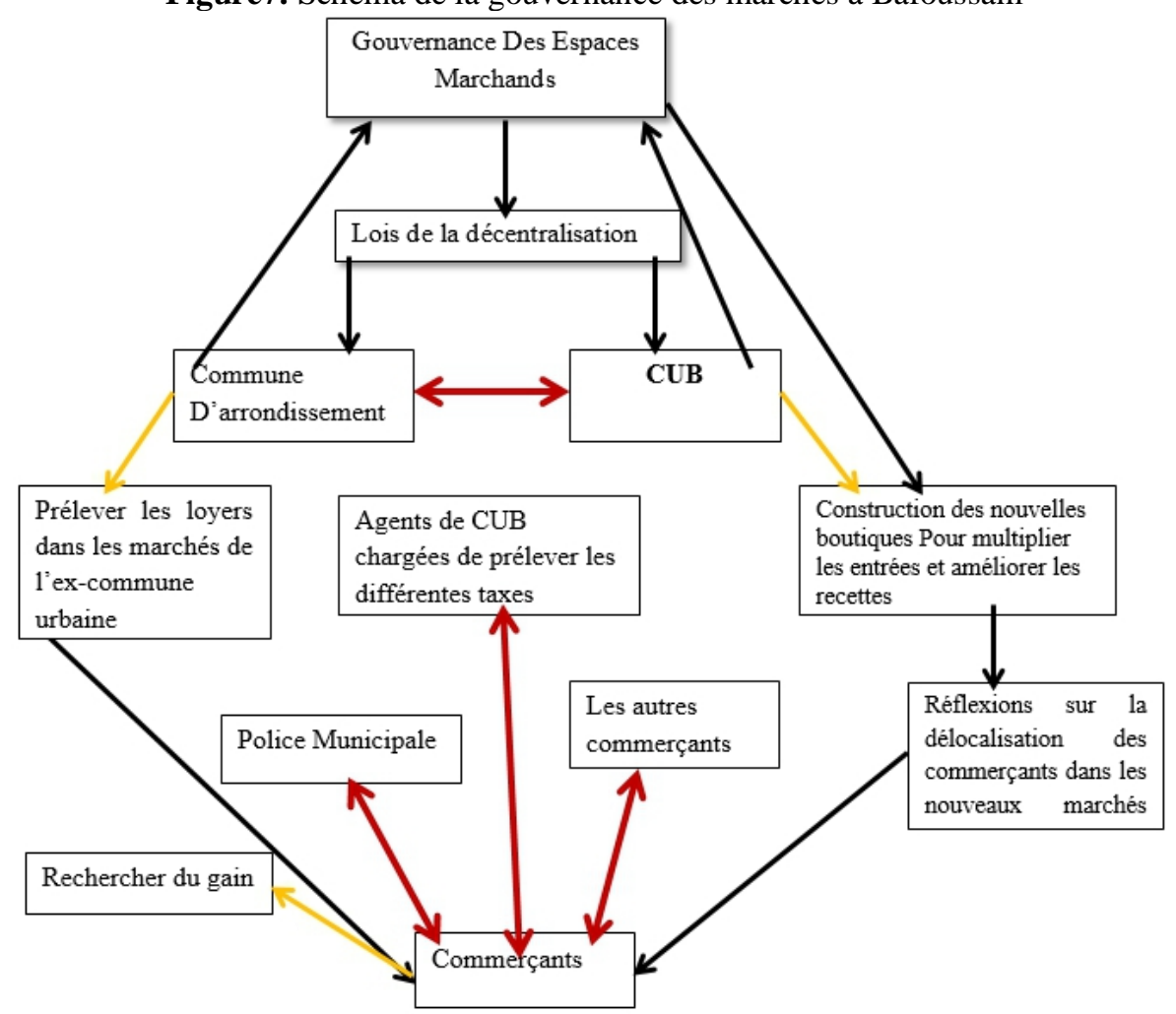

Légende :

Yemele, 2017

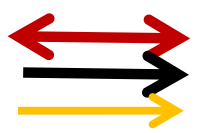

Rapport conflictuel d'opposition Rapport de collaboration Rapport d'intérêts

Ce schéma a permis de présenter les résultats de la gouvernance des espaces marchands à Bafoussam. Il est donc clair que la CUB, les communes d'arrondissements et les commerçants recherchent des profits immesurables et c'est de là que naissent les conflits. Il s'avère également nécessaire 
d'engager des réflexions, des concertations sur la gestion des espaces marchands. Des modes de gouvernance à l'œuvre autour des marchés devraient être déjà revus et s'orienter vers une gouvernance plus inclusive. Alors à travers l'analyse de $\mathrm{SWOT}^{12}$, nous avons pu relever les forces, les opportunités, les faiblesses et les menaces résumés dans le tableau ci-dessous :

Tableau1. L'analyse SWOT sur la gouvernance des espaces marchands

\begin{tabular}{|c|c|}
\hline Forces & Faiblesses \\
\hline $\begin{array}{l}\text {-Concentrer sur l'aménagement et l'organisation } \\
\text { des espaces marchands } \\
\text {-Faire intervenir tous les acteurs dans la gestion }\end{array}$ & $\begin{array}{l}\text {-Lois existantes peu claires } \\
\text {-Peu de liens avec les autres acteurs } \\
\text {-Désaccord sur les modes de gouvernance avec les } \\
\text { autres gestionnaires }\end{array}$ \\
\hline Opportunités & Menaces \\
\hline $\begin{array}{l}\text {-Intérêts individuel et publique croissant sur } \\
\text { l'amélioration des revenus } \\
\text {-Réglementation gouvernementale visant à } \\
\text { redéfinir le rôle de chaque acteur } \\
\text {-Développement de nouveaux marchés dans le } \\
\text { but de changer la qualité des infrastructures et le } \\
\text { visage de la ville }\end{array}$ & $\begin{array}{l}\text {-Influence des élus locaux sur les commerçants (à } \\
\text { propos de la collecte des loyers et diverses taxes) } \\
\text {-Nouvelle législation pour la protection des } \\
\text { commerçants n'ayant pas de boutiques ou d'étals }\end{array}$ \\
\hline
\end{tabular}

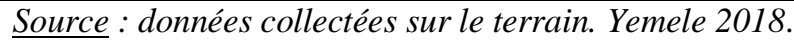

$\mathrm{Au}$ vu de ce tableau, nous constatons que cette analyse permet de créer des stratégies pertinentes pour bien développer et organiser les espaces marchands, d'anticiper une menace pesant sur l'espace d'activité.

\section{III- Discussion}

La gouvernance urbaine dans les espaces marchands à Bafoussam avait pour but de rehausser le visage de la ville. Mais son application a favorisé la présence de plusieurs acteurs dans les marchés et gares routières entrainant la recherche démesurée du profit et par conséquent la naissance des conflits. Les enjeux relevés sont d'ordre socio-économique (source de recette permettant d'améliorer le chiffre d'affaire de la CUB et la recherche des revenu pour les commerçants), fonciers (les commerçants démontrent une volonté de garantir le contrôle et la possession de leur place de vente), et écologiques (L'environnement des espaces marchands est caractérisé par l'insalubrité, la promiscuité, l'inconfort et l'accessibilité difficile). Le questionnement reste au niveau du scénario de gouvernance urbaine qui concilie tous les intérêts divergents. Les travaux de Bagnosco \& al (1997) complète en soutenant que la coopération et l'accommodement entre des intérêts divers et conflictuels est un processus continu. Dès lors, la construction et le réaménagement des boutiques dans les marchés et tout long de la ville se présentent comme une option permettant de satisfaire la demande

${ }^{12}$ Strengths - Weaknesses - Opportunities - Threats ou MOFF pour les Francophones : Menaces - Opportunités - Forces - Faiblesses 
et de stimuler les activités économiques. Seulement ces aménagements et l'organisation des marchés sont déclencheurs de conflits.

Ces politiques de gouvernance sur les espaces marchands seront susceptibles de réduire l'occupation anarchique dans la ville. Elles réguleraient la pression foncière observée. Cette tendance provoque des changements et pose des défis majeurs auxquels, il est aujourd'hui important de trouver des solutions : une demande croissante en équipements et en services urbains de base (eau, assainissement, électricité, ect...), Noundjeu (2006). Cette réalité massive et multiforme accentue ainsi un processus de décomposition-recomposition urbaine.

Il s'avère alors nécessaire que la ville soit placée au cœur des questions de développement, de la recherche urbaine et des projets, et que des relais nouveaux soient créés tant dans l'élaboration que dans la mise en œuvre des politiques urbaines, au travers des $\mathrm{ONG}$, des associations.

$\mathrm{Vu}$ ce qui précède, la complexité du problème de gouvernance qui se pose dans les espaces marchands de Bafoussam n'est-elle pas à priori liée à la mentalité des différents acteurs? Cette gouvernance ne se présente-t-elle pas comme étant la logique la plus crédible face aux multiples enjeux et conflits qui interfèrent en continu ?

\section{Conclusion}

A la lumière de ce qui précède, l'on remarque la place qu'occupent les espaces marchands dans les pays africains en général et dans la ville de Bafoussam en particulier à travers son attractivité et les flux de transaction qu'il engendre. Ils constituent un domaine privilégié pour l'application des principes de la gouvernance. Ces principes ont du mal à être appliqués à cause d'une part de la recherche démesurée des profits par les acteurs (Communauté Urbaine, Commune d'Arrondissement, commerçants) et d'autre part de la non maitrise des différents rôles joués par ces derniers. Dans ce contexte, la réglementation et la coordination des espaces marchands deviennent difficiles. Par conséquent, on assiste à des chevauchements de compétence, conflit lié à l'augmentation des impôts et a de multiples résistances. Pour gérer ces conflits, ce processus peut alors être accompagné par la mise en œuvre d'une intermédiation sociale. Ce mécanisme vise à responsabiliser l'ensemble des parties en essayant de les amener à une négociation permettant à chacune d'elles d'arriver à un compromis. Bien que limité dans le temps, ce type de cellule doit être vu comme un outil. Son existence doit être prolongée, sous une forme plus intégrée au processus de dialogue local pour faciliter les conflits de gestion qui ne manqueront pas de se poser.

Nous pouvons dire que les défis auxquels le territoire africain en particulier le Cameroun se heurte, devraient être relevés dans les années à venir. Une politique d'aménagement devra se déployer autour de nouveaux 
défis : enjeux urbain, environnemental, économique, social, enjeu de gouvernance, enjeu institutionnel et régional.

\section{References :}

1. Assako, R. J., \& Ndock, G., (2010). Gouvernance urbaine et développement local dans la Commune de Soa à la périphérie Nord de Yaoundé - Cameroun. Annales de la Faculté des Arts, Lettres et Sciences Humaines de l'Université de Ngaoundéré, Vol. XII, pp 185 212.

2. Dongmo Tsobzé A., Minla, J., \& Hilhorst T., (2007). Entre désespoir et espoir: Les défis de la gouvernance et de la décentralisation dans la réalisation des investissements publics locaux au Cameroun. KIT \& SNV Cameroun, Yaoundé.

3. Binam, D. (2015). Gouvernance urbaine au Cameroun : une analyse socio-anthropologique du jeu et des enjeux des acteurs dans la gestion urbain. Mémoire de Master 2, Université de Yaoundé II, 166p.

4. Noundjeu, F. C. (2006). Impact de la décentralisation sur la Gouvernance urbaine au Cameroun : Cas de la ville de Bangangté. 24p.

5. Gaëlle, H., \& Poisbeau, F. (2015). L'AFD \& et les équipements urbains marchands : 30 ans de projets de réhabilitation de marchés en Afrique, www.afd.fr

6. Hatcheu, E., Yemmafouo, A., Nzomo, J., Lemougoue, J. (2009). Enjeux et rivalité autour de la gestion des espaces urbains dans une ville moyenne d'Afrique centrale: les marchés et les gares routières à Dschang.

7. Hatcheu, E. (2005).Espaces marchands et gouvernance urbaine en Afrique : l'exemple de douala au Cameroun. Communication au quatrième Journée scientifique du comité nationale de géographie du Cameroun, Douala.

8. Jaglin, S. (2007). Décentralisation et gouvernance de la diversité. Les services urbains en Afrique anglophone. Op.cit. p.31

9. Jouve, B., \& Lefevre, C. (1999). De la gouvernance urbaine au gouvernement des villes? Permanence ou recomposition des cadres de l'action publique en Europe. Revue Française de Science Politique, vol. $49, n^{\circ} 6$, p. 835

10. Kengne F., (1991) Les petits métiers de rue et l'emploi, le cas de Yaoundé, Ed sopecam, collections idées.163 p

11. Le Bris, E. (2008). Gouvernance urbaine dans tous ses états, Ministère des Affaires étrangères et européennes, DGCID, France.

12. Le Galès, P. (1995). Du Gouvernement des villes à la gouvernance urbaine. Revue Française de Science Politique, vol. 45, Pp5792 
13. Le Galès, P. (1997). Gouvernement et Gouvernance des régions : faiblesses structurelles et nouvelles mobilisations, in Le Galès(P.), (dir.), Les paradoxes des régions en Europe, Paris, La Découverte, p.130

14. Michelon, B. (2008). La gouvernance dans les projets d'équipements marchands en Afrique : Gouvernement et gouvernance des espaces urbains. http//Calenda revues.org/nouvelle10961.html.

15. Ndoki, D., Mofor, G. \& Kankeu, R. (2016). La Délocalisation des Gares Routières comme Moyen de Désengorgement du Centre Urbain de Bafoussam (Ouest-Cameroun).

16. Perrick, L. (2008). Le marché urbain : intervention de développement et modes d'appropriation par les acteurs locaux, Le cas du Grand Marché et du Marché aux légumes à Ouahigouya (Burkina Faso), 95p

17. Pacaud, P.A., \& Filippi. (2004) Rénover les marchés urbains : L'exemple de Bobo-Dioulasso.

18. Pinson G. (2009). Gouverner les villes par projets. Presse de Science Po, Paris.

19. Poirot, J. (2005). Le modèle européen de ville durable, Urbanisation, Gouvernance et Développement Durable. Cahiers de Préludes n ${ }^{\circ}$, pp. (13-47)

20. Wilhelm, L. (1994). Les circuits d'approvisionnement et le fonctionnement des marchés en Afrique et à Madagascar, synthèse des études socio-économiques récentes.

21. Yemele, F. (2011). Production et gestion des espaces marchands dans la ville de Dschang (Ouest-Cameroun).

22. Yemmafouo, A. (2018) Street vending Power Relationships and Governance of Public Spaces in Bafoussam, West Cameroon. Current Urban Studies, 6,611-629. Doi: 10.4236/cus.2018.64032.

23. Loi $\mathrm{n}^{\circ}$ 90-031 du 10 Août 1990 régissant l'activité commerciale au Cameroun.

24. Loi n²009/019 du 15 décembre 2009 portant fiscalité locale.

25. Loi $n^{\circ}$ 04-018 du 22 juillet 2004 fixant les règles applicables aux communes.

26. Décret $\mathrm{N}^{\circ}$ 2015/1375/PM du 08 Juin 2015 fixant les modalités d'exercice de certaines compétences transférées par l'Etat aux Communes en matière de commerce.

27. Décret no 2008/025 du 17 Janvier portant création de la Communauté Urbaine de Bafoussam, In Cameroon Tribune, no 9018 du vendredi 18 Janvier 2008, p5. 